\title{
Population Synthesis of Old Neutron Stars in the Galaxy
}

\author{
S. B. Popov
}

Sternberg Astronomical Institute, Universiteskii Pr. 13, 119899, Moscow, Russia

\section{Colpi}

Dept. of Physics, Univ. of Milan, Via Celoria 16, 20133 Milan, Italy

\section{A. Treves}

Dipartimento di Scienze, Univ. dell'Insubria, Via Lucini 3, 22100, Como, Italy

\section{R. Turolla}

Dept. of Physics, Univ. of Padova, Via Marzolo 8, 35131 Padova, Italy

\section{M. Lipunov}

Dept. of Physics, Moscow State Univ., Sternberg Astronomical Institute, Universiteskii Pr. 13, 119899, Moscow, Russia

\section{E. Prokhorov}

Sternberg Astronomical Institute, Universiteskii Pr. 13, 119899, Moscow, Russia

\begin{abstract}
The paucity of old, isolated accreting neutron stars in ROSAT observations is used to derive a lower limit on the mean velocity of neutron stars at birth. The secular evolution of the population is simulated following the paths of a statistical sample of stars for different values of the initial kick velocity, drawn from an isotropic, Gaussian distribution with mean velocity $0 \leq\langle V\rangle \leq 550 \mathrm{~km} \mathrm{~s}^{-1}$. The spin-down, induced by dipole losses and the interaction with the ambient medium, is tracked together with the dynamical evolution in the Galactic potential, allowing for the determination of the fraction of stars which are, at present, in each of the four possible stages: Ejector, Propeller, Accretor, and Georotator. Taking from the ROSAT All-Sky Survey an upper limit of $\sim 10$ accreting neutron stars within $\sim 140 \mathrm{pc}$ from the Sun, we infer a lower bound for the mean kick velocity, $\langle V\rangle \gtrsim 200-300 \mathrm{~km} \mathrm{~s}^{-1}$. The same conclusion is reached for both a constant $\left(B \sim 10^{12} \mathrm{G}\right)$ and an exponentially decaying magnetic field with a timescale $\sim 10^{9} \mathrm{yr}$. Present results, moreover, constrain the fraction of low-velocity stars which could have escaped pulsar statistics to $\lesssim 1 \%$.
\end{abstract}




\section{Introduction}

Isolated neutron stars (NSs) are expected to be as many as $10^{8}-10^{9}$, a nonnegligible fraction of the total stellar content of the Galaxy. The number of observed radio pulsars is now $\sim 1,000$. Since the pulsar lifetime is $\sim 10^{7} \mathrm{yr}$, this implies that the bulk of the NS population, mainly formed of old objects, remains undetected as yet. Despite intensive searches at all wavelengths, only a few (putative) isolated NSs which are not radio pulsars (or soft $\gamma$ repeaters) have been recently discovered in the X-rays with ROSAT (Walter, Wolk, \& Neuhauser 1996; Haberl, Motch, \& Pietsch 1998; Neuhauser \& Trumper 1999). The extreme X-ray to optical flux ratio $\left(>10^{3}\right)$ makes the NS option rather robust, but the exact nature of their emission is still controversial. Up to now, two main possibilities have been suggested, either relatively young NSs radiating away their residual internal energy or much-aged NSs accreting the interstellar medium (ISM). Both options have advantages and drawbacks. Standard cooling atmosphere models fail to predict in a natural way the spectrum of the best studied object, RX J1856-3754 (see Walter et al. 2000, these Proceedings). Accretion models require instead a very low NS velocity relative to the ISM ( $v<$ $20 \mathrm{~km} \mathrm{~s}^{-1}$ ) in order to produce the luminosities inferred from ROSAT data (see Walter et al. 2000, these Proceedings). We feel that a more thorough analysis of the statistical properties of NSs can be useful in providing indirect evidence in favor or against the accretion scenario.

As discussed by Lipunov (1992), isolated NSs can be classified into four main types: Ejectors, Propellers, Accretors, and Georotators. In Ejectors, the relativistic, outflowing momentum flux is always larger than the ram pressure of the surrounding material, so they never accrete and are either active or dead pulsars, still spun down by dipole losses. In Propellers, the incoming matter can penetrate down to the Alfvén radius, $R_{A}$, but no further because of the centrifugal barrier, and stationary inflow can not occur, but the piling up of the material at the Alfvén radius may give rise to (supposedly short) episodes of accretion (Treves, Colpi, \& Lipunov 1993; Popov 1994). Steady accretion is also impossible in Georotators where (similarly to the Earth) the Alfvén radius exceeds the accretion radius so that magnetic pressure dominates everywhere over the gravitational pull. It is the combination of the stellar period, magnetic field, and velocity that decides which type a given isolated NS belongs to, and, since both $P, B$, and $V$ change during the star's evolution, an NS can go through different stages in its lifetime.

While the dynamical evolution of NSs in the Galactic potential was studied by several authors (see, e.g., Madau \& Blaes 1994; Zane et al. 1995), little attention was paid to the NSs' magneto-rotational evolution. Recently, this issue was discussed in some detail by Livio, Xu, \& Frank (1998) and Colpi et al. (1998). The goal of this investigation is to consider these two issues simultaneously, coupling the dynamical and the magneto-rotational evolution for the isolated NS population.

The possibility that the low-velocity tail is underpopulated with respect to what was previously assumed should be seriously taken into account. It is our aim to revise the estimates on the number of old, accreting neutron stars in the Galaxy in the light of these new data, in the attempt to reconcile theoretical predictions with present ROSAT limits (Neühauser \& Trümper 1999). 


\section{The Model}

In this section, we summarize the main hypotheses introduced to track the evolution of single stars and describe shortly the technique used to explore their statistical properties, referring to Popov \& Prokhorov (1998) for details on spatial evolution calculations and to Konenkov \& Popov (1997) and Lipunov \& Popov (1995) for details of magneto-rotational evolution.

\subsection{Dynamical Evolution}

The dynamical evolution of each single star in the Galactic potential (taken in the form proposed by Miyamoto \& Nagai 1975) is followed solving its equations of motion.

The period evolution depends on both the stellar velocity and the local density of the interstellar medium; any attempt to investigate the statistical properties of the NS population should incorporate a detailed model of the ISM geography. Unfortunately, the distribution of molecular and atomic hydrogen in the Galaxy is highly inhomogeneous. Here, we use the analytical distributions from Bochkarev (1992) and Zane et al. (1995) for the hydrogen density $n(R, Z)$. Within a region of $\sim 140 \mathrm{pc}$ around the Sun, the ISM is underdense, and we take $n=0.07 \mathrm{~cm}^{-3}$.

In our model, we assume that the NS birthrate is constant in time and proportional in magnitude to the square of the local gas density.

Neutron stars at birth have an orbital velocity determined by the Galactic potential. Superposed to this ordered motion a kick velocity is imparted in a random direction. We use here an isotropic, Gaussian distribution (relative to the local orbital speed), with dispersion $\sigma_{V}$, simply as a means to model the true pulsar distribution at birth (see, e.g., Cordes \& Chernoff 1998). The mean velocity $\langle V\rangle=(8 / \pi)^{1 / 2} \sigma_{V}$ is varied in the interval $0-550 \mathrm{~km} \mathrm{~s}^{-1}$.

\subsection{Accretion Physics and Period Evolution}

The accretion rate was calculated according to the Bondi formula,

$$
\dot{M}=\frac{2 \pi(G M)^{2} m_{p} n(R, Z)}{\left(V^{2}+V_{s}^{2}\right)^{3 / 2}} \simeq 10^{11} n v_{10}^{-3} \mathrm{~g} \mathrm{~s}^{-1}
$$

where $m_{p}$ is proton mass, the sound speed $V_{s}$ is always $10 \mathrm{~km} \mathrm{~s}^{-1}$, and $v_{10}=$ $\left(V^{2}+V_{s}^{2}\right)^{1 / 2}$ in units of $10 \mathrm{~km} \mathrm{~s}^{-1} . M$ and $R$ denote the NS mass and radius, which we take equal to $1.4 M_{\odot}$ and $10 \mathrm{~km}$, respectively, for all stars.

All neutron stars are assumed to be born with a period $P(0)=0.02 \mathrm{~s}$ and a magnetic moment, either $\mu_{30}=1$ or $\mu_{30}=0.5$, where $\mu_{30}=\mu / 10^{30} \mathrm{G} \mathrm{cm}^{3}$.

In the Ejector phase, the energy losses are due to magnetic dipole radiation. When the gravitational energy density of the incoming interstellar gas exceeds the outward momentum flux at the accretion radius, $R_{a c} \simeq 2 G M / v^{2}$, matter starts to fall in. This happens when the period reaches the critical value

$$
P_{E}(E \rightarrow P) \simeq 10 \mu_{30}^{1 / 2} n^{-1 / 4} v_{10}^{1 / 2} \mathrm{~s} .
$$

When $P>P_{E}(E \rightarrow P)$, the NS is in the Propeller phase, rotational energy is lost, and the period keeps increasing at a rate taken from Shakura (1975). 

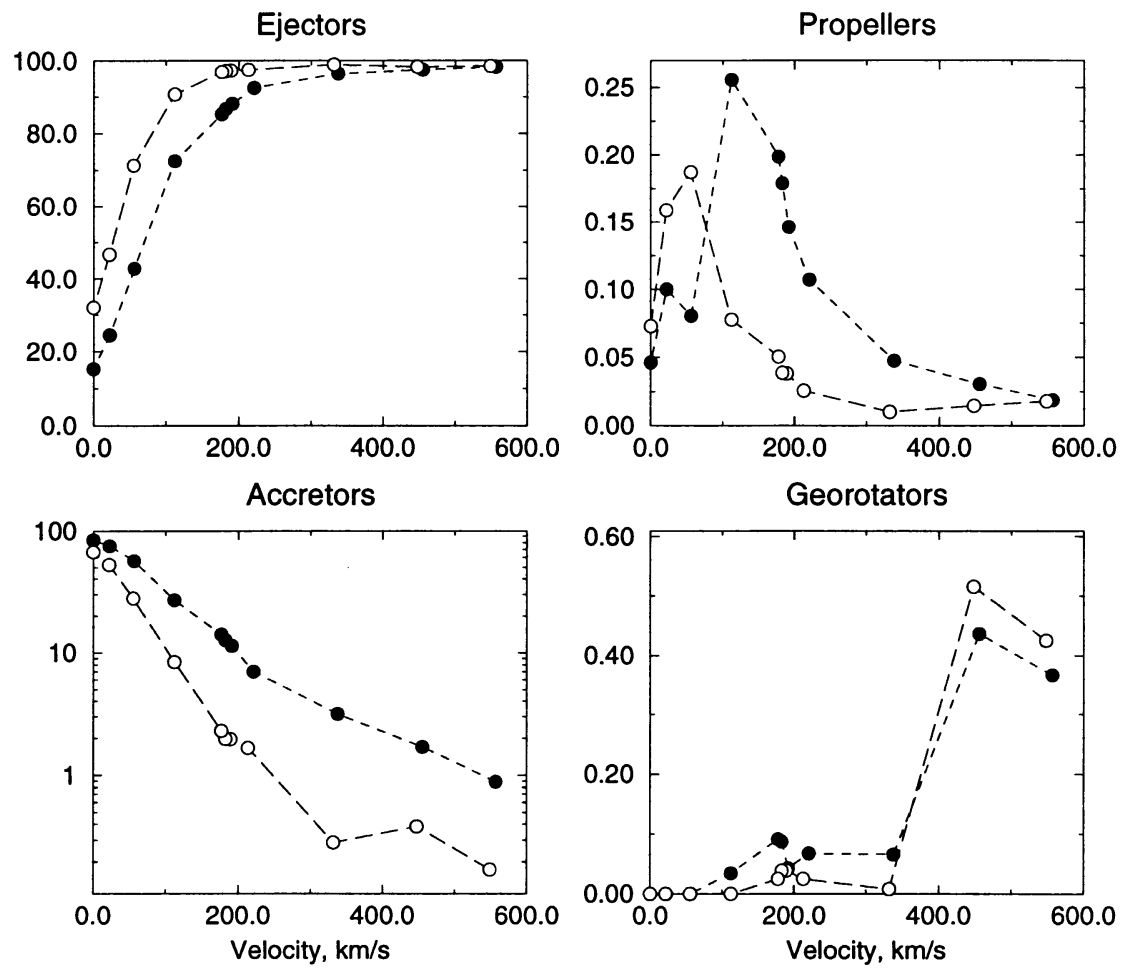

Figure 1. Fractions of NSs (in percents) in the different stages vs. the mean kick velocity for $\mu_{30}=0.5$ (open circles) and $\mu_{30}=1$ (filled circles); typical statistical uncertainty for ejectors and accretors is $1-2 \%$.

As the star moves through the inhomogeneous ISM, a transition from the Propeller back to the Ejector phase may occur if the period attains the critical value

$$
P_{E}(P \rightarrow E) \simeq 3 \mu_{30}^{4 / 5} v_{10}^{6 / 7} n^{-2 / 7} \mathrm{~s} .
$$

Note that the transitions $P \rightarrow E$ and $E \rightarrow P$ are not symmetric as first discussed by Shvartsman in the early '70s.

Accretion onto the stellar surface occurs when the corotation radius $R_{c o}=$ $\left(G M P^{2} / 4 \pi^{2}\right)^{1 / 3}$ becomes larger than the Alfvén radius (and $R_{A}<R_{a c}$, see below). This implies that braking torques have increased the period up to

$$
P_{A}(P \rightarrow A) \simeq 420 \mu_{30}^{6 / 7} n^{-3 / 7} v_{10}^{9 / 7} \mathrm{~s} .
$$

As soon as the NS enters the Accretor phase, torques produced by stochastic angular momentum exchanges in the ISM slow down the star's rotation to the equilibrium period

$$
P_{e q}=2.6 \times 10^{3} v_{t, 10}^{-2 / 3} \mu_{30}^{2 / 3} n^{-2 / 3} v_{10}^{13 / 3} \mathrm{~s},
$$




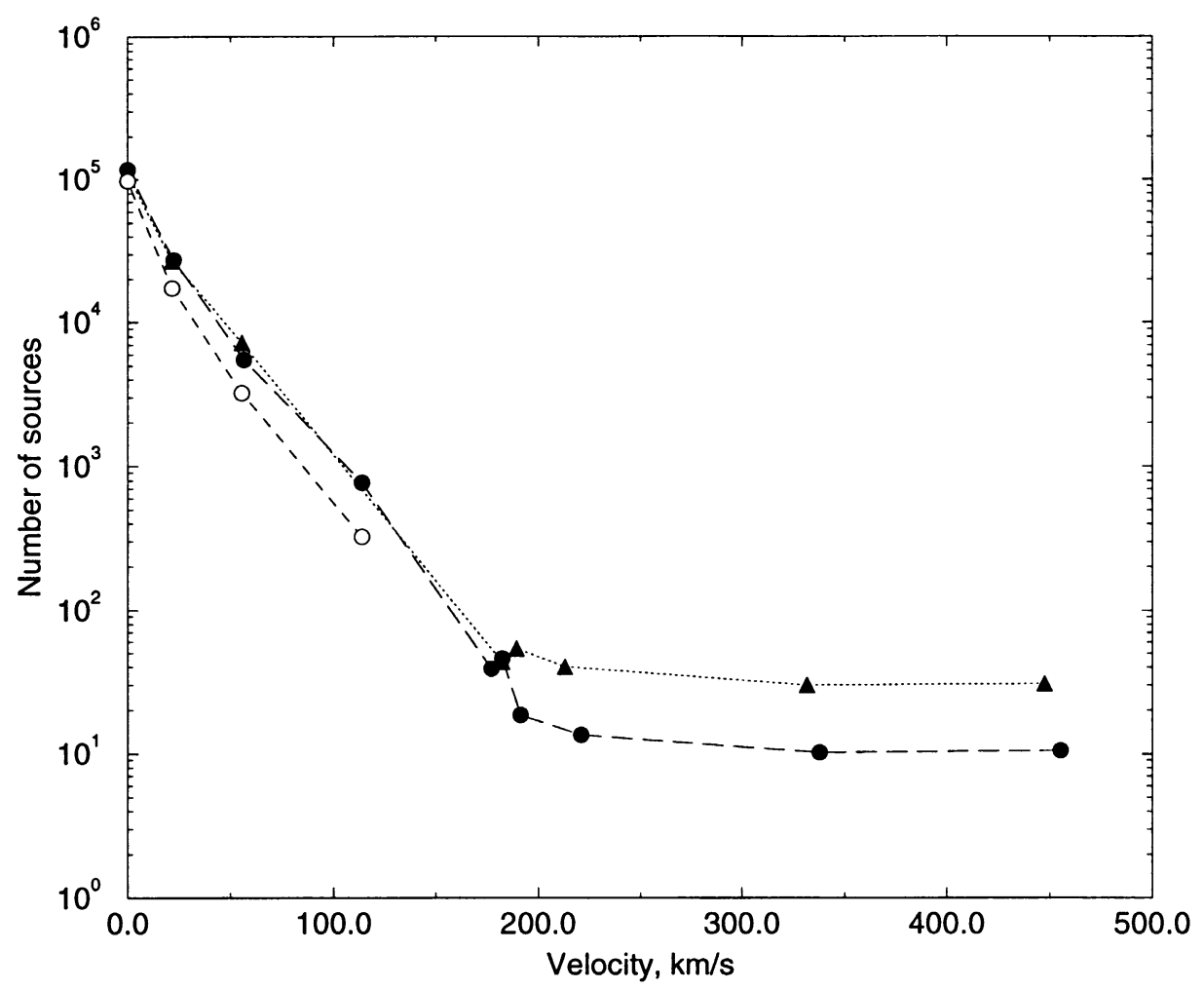

Figure 2. Number of accreting NSs in the Solar vicinity for a constant ( $\mu_{30}=1$, filled circles; $\mu_{30}=0.5$, opened circles) and decaying $\left(t_{d}=\right.$ $2.2 \times 10^{9} \mathrm{yr}$, triangles) field.

where $v_{t}$ is the turbulent velocity of the ISM (Lipunov \& Popov 1995; Konenkov \& Popov 1997).

At the very low accretion rates expected for fast, isolated NSs, it could be that the Alfvén radius is larger than the accretion radius. The condition $R_{A}<R_{a c}$ translates into a limit for the stellar velocity:

$$
v<410 n^{1 / 10} \mu_{30}^{-1 / 5} \mathrm{~km} \mathrm{~s}^{-1} .
$$

\section{Results and Discussion}

\subsection{The NS Census for a Non-Decaying Field}

We consider two representative values for the (constant) magnetic dipole moment, $\mu_{30}=0.5$ and $\mu_{30}=1$. The present fraction of NSs in the Ejector and Accretor stages as a function of the mean kick velocity is shown in Figure 1.

Here, and in the following, the total number of Galactic NSs was assumed to be $10^{9}$. A total number $\sim 10^{9}$ appears to be consistent with the nucleosynthesis and chemical evolution of the Galaxy, while $10^{8}$ is derived from radio pulsars observations. It is uncertain if all NSs experience an active radio pulsar phase, 

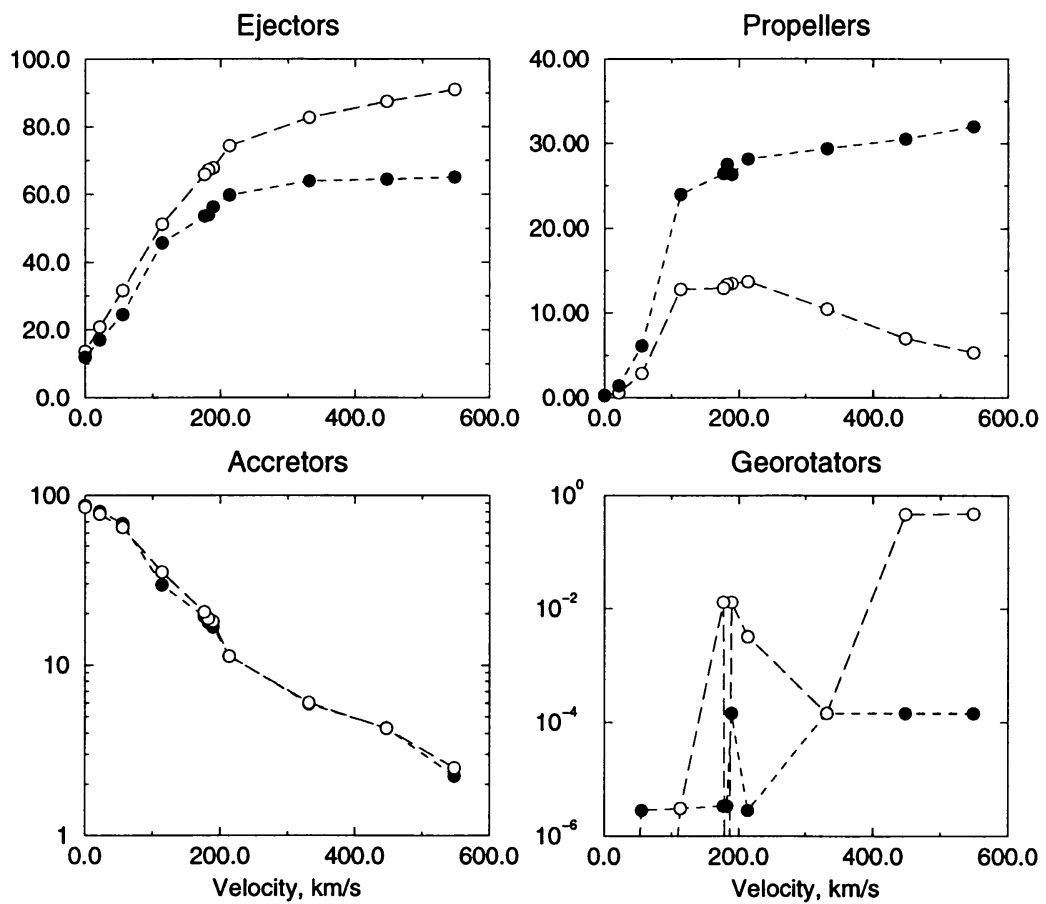

Figure 3. Fractions of NSs (in percents) in the different stages vs. the average kick velocity for a decaying field with an e-folding time $t_{d}=2.2 \times 10^{9} \mathrm{yrs}$ (open circles) and $t_{d}=1.1 \times 10^{9} \mathrm{yrs}$ (filled circles).

due to low initial magnetic fields, long periods, or the fall-back in the aftermath of the supernova explosion. There is a serious possibility that the total number of NSs derived from radio pulsar statistics is only a lower limit.

In order to compare the expected number of accreting "Old NSs" with the ROSAT All-Sky Survey (RASS) results, we evaluated the number of those ONSs, within $140 \mathrm{pc}$ from the Sun, producing an unabsorbed flux of $10^{-13} \mathrm{erg} \mathrm{cm}^{-2} \mathrm{~s}^{-1}$ or higher at energies $\sim 100 \mathrm{eV}$. The results are illustrated in Figure 2. The main point is that for mean velocities below $200 \mathrm{~km} \mathrm{~s}^{-1}$ the number of ONSs with a flux above the RASS detection limit would exceed 10. The most recent analysis on the number of isolated NSs in the RASS (Neühauser \& Trümper 1999 ) indicates that the upper limit is below 10.

An important aspect is that our results exclude the possible presence of a consistent low-velocity population at birth, which exceeds that contained in the Gaussian with $\langle V\rangle>200 \mathrm{~km} \mathrm{~s}^{-1}$.

\subsection{The NS Census for a Decaying Field}

We refer here only to a very simplified picture of the field decay in which $B(t)=$ $B(0) \exp \left(-t / t_{d}\right)$. Calculations have been performed for $t_{d}=1.1 \times 10^{9} \mathrm{yr}, t_{d}=$ $2.2 \times 10^{9} \mathrm{yr}$, and $\mu_{30}(0)=1$. Results are shown in Figure 3 . 


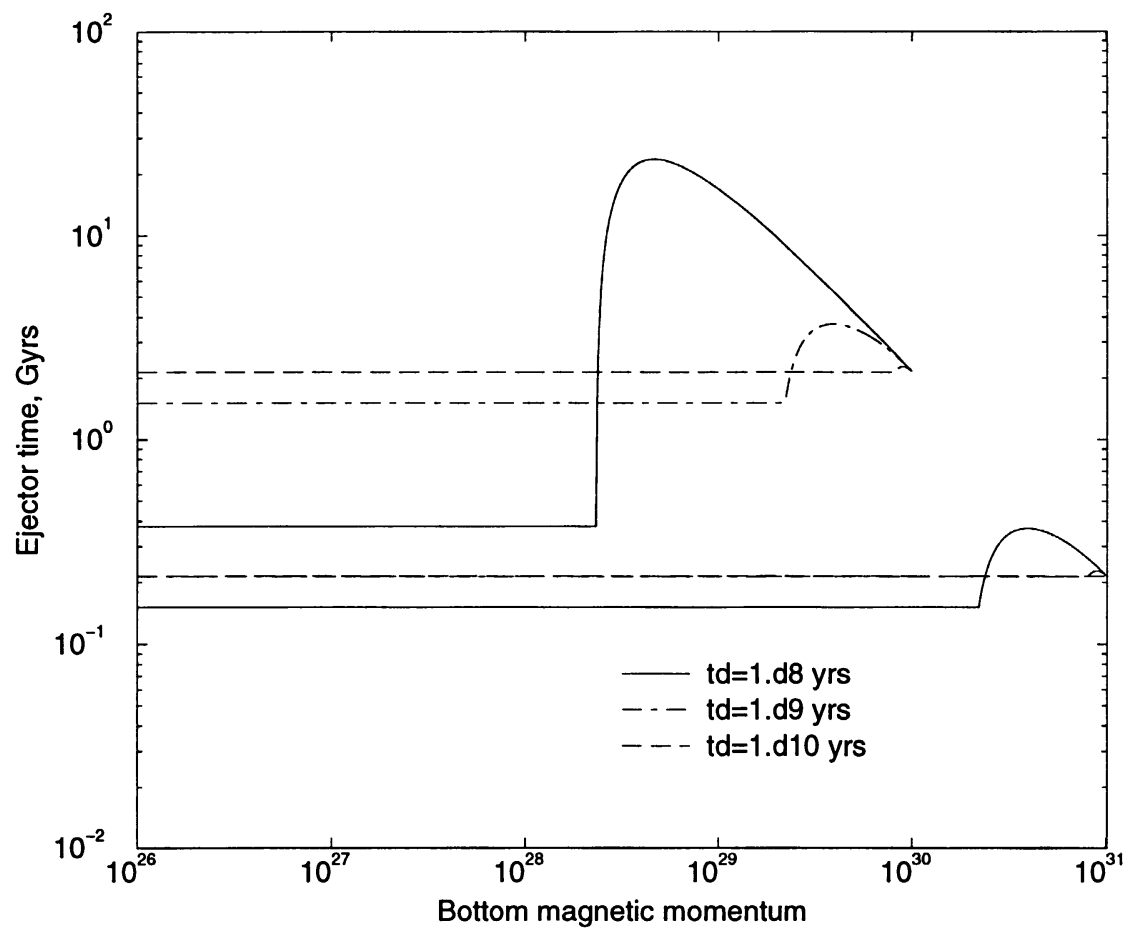

Figure 4. Ejector time vs. bottom magnetic momentum for two values of initial magnetic momentum and different decay timescales, $t_{d}=10^{8}, 10^{9}$, and $10^{10} \mathrm{yr}$.

For some values of $t_{d}$ and bottom field, most NSs can stay at the Ejector stage, and the number of Accretors and Propellers would not be increased. We show these analytical estimates graphically in Figure 4, where the Ejector time, $T_{E}$, is plotted vs. bottom magnetic momentum for constant velocity and ISM density $\left(n=1 \mathrm{~cm}^{-3}, v=10 \mathrm{~km} / \mathrm{s}\right)$, different $t_{d}$, and two values of the initial magnetic momentum, $10^{30}$ and $10^{31} \mathrm{G} \mathrm{cm}^{3}$ (see Popov \& Prokhorov 2000).

Summarizing, we can conclude that, although both the initial distribution and the subsequent evolution of the magnetic field strongly influences the NS census and should be accounted for, the lower bound on the average kick derived from ROSAT surveys is not very sensitive to $B$, at least for not too extreme values of $t_{d}$ and $\mu(0)$, within this model.

\section{Conclusions}

In this paper, we have investigated how the present distribution of neutron stars in the different stages (Ejector, Propeller, Accretor, and Georotator) depends on the star's mean velocity at birth. On the basis of a total of $\sim 10^{9} \mathrm{NSs}$, the fraction of Accretors was used to estimate the number of sources within 
$140 \mathrm{pc}$ from the Sun which should have been detected by ROSAT. The most recent analysis of ROSAT data indicates that no more than $\sim 10$ non-optically identified sources can be accreting ONSs. This implies that the average velocity of the NS population at birth has to exceed $\sim 200 \mathrm{~km} \mathrm{~s}^{-1}$, a figure which is consistent with those derived from radio pulsars statistics. We have found that this lower limit on the mean kick velocity is substantially the same either for a constant or a decaying $B$-field, unless the decay timescale is shorter than $\sim 10^{9} \mathrm{yr}$. Since observable accretion-powered ONSs are slow objects, our results exclude also the possibility that the present velocity distribution of NSs is richer in low-velocity objects with respect to a Maxwellian. The paucity of accreting ONSs seem, therefore, to lend further support in favor of neutron stars as very fast objects.

Acknowledgments. This work was partially supported by the European Commission under contract ERBFMRX-CT98-0195. The work of S.P., V.L and M.P. was supported by grants RFBR 98-02-16801 and INTAS 96-0315. S.P. and V.L. gratefully acknowledge the University of Milan and of Insubria (Como) for support during their visits. S.P. also acknowledges the organizers of IAU 195.

\section{References}

Bochkarev, N. G. 1992, Basics of the ISM Physics (Moscow: Moscow University Press)

Colpi, M., Turolla, R., Zane, S., \& Treves, A. 1998, ApJ, 501, 252

Cordes, J. M., \& Chernoff, D. F. 1998, ApJ, 505, 315

Haberl, F., Motch, C., \& Pietsch, W. 1998, Astron. Nachr., 319, 97

Konenkov, D. Yu., \& Popov, S. B. 1997, PAZh, 23, 569

Lipunov, V. M. 1992, Astrophysics of Neutron Stars (Berlin: Springer \& Verlag)

Lipunov, V. M., \& Popov, S. B. 1995, AZh, 71, 711

Livio, M., Xu, C., \& Frank, J. 1998, ApJ, 492, 298

Madau, P., \& Blaes, O. 1994, ApJ, 423, 748

Miyamoto, M., \& Nagai, R. 1975, Pub. Astr. Soc. Japan, 27, 533

Neühauser, R., \& Trümper, J. E. 1999, A\&A, 343, 151

Popov, S. B. 1994, Astron. Circ., N1556, 1

Popov, S. B., \& Prokhorov, M. E. 1998, A\&A, 331, 535

Popov, S. B., \& Prokhorov, M. E. 2000, A\&A, submitted

Shakura, N. I. 1975, PAZh, 1, 23

Treves, A., Colpi, M., \& Lipunov, V. M. 1993, A\&A, 269, 319

Walter, F. M., Wolk, S. J., \& Neühauser, R. 1996, Nature, 379, 233

Walter, F. M., An, P., Lattimer, J., \& Prakash, M. 2000, these Proceedings

Zane, S., Turolla, R., Zampieri, L., Colpi, M., \& Treves, A. 1995, ApJ, 451, 739 